en rapport avec la question de l'aménagement des eaux aux notions au moins fondamentales, mais acquises pratiquement, sur le terrain même, nécessaires pour mener à bien cet aménagement. Ainsi saura-ton pratiquement quand une eau polluée, puis épurée, sera redevnue zoïque; si un endroit classique de déversement ronvient aussi par sa sitèse ; par conséquent, s'il le faut conserver ou modificr.

Ici s'imposent les laboratoires volants, si cn vogue en Imérique par exemple.

Programme économique. - Transformer l'aquiculture et la pisciculture en branches rationuelles dı savoir humain, et les dégager d'un empirisme qui a trop longlemps ven. Berhercher les causes scientifiques des dépeuplements et les moỵns scientifiques d'y remédier. Favoriser les recherches d'irhthyopathologie trop négrigées parfois.

\title{
LES TRAVAUX DE LA STATION YOUGOSLAVE DE RECHERCHES CARPICOLES DURANT LES CINQ PREMIËRES ANNÉES
}

\author{
Par M. le Professeur Docteur E. RÖSSLER
}

do Mniversit: de Zagreb, Institut do \%oologic applique: (1).

Dès igıf. W. Zwrtave propriétaire d'étangs à Zdencina (Croatie). fit, aux autorités provinciales l'alors, l'offre de créer à ses frais, dans son domaine, une station de recherehes carpicoles, pour fonctionner souts lit direction de l'toministration. Walheureusement, les rirconstances anormales qui prévalurent durant la guerre of les annés consécutives mirent obstacle à la réalisation de ce projet. I’installation des viviers of l'aménagement des lieux s'en trousèrent retardés. C'est seulement en rgoj, une fois surmontées des difficultés variées, que l'étahlissement, rattaché à l'Institut de zoologrie appliqués dont le siège est à Zagreb, put commencer ses I ravaux.

La Station dispose de six étangs de recherches d'une superficie grlobale de I Hectare 7 ares, six aulres ayant dû it re supprimés comme inutilisitbles. Ils sont situés au centre du domaine, dans la vaste plaine au sud de la chaîne de Plejsivica, à environ in kilomètres de la gare de Zdencina, sır la ligne de Zagreb à Fiume.

Les viviers ont chacum leur alimentation séparée et empruntent leur eau

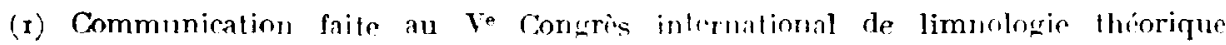
et appliquée, à Burrapest, en Aồt ı.3o. 
à une dérivation de la rivière Okisnica qui, par ailleurs, approvisionne nresque tous les étangs du domaine de M. Zwilling.

A proximité de ces viviers, au premier étage d'un bâtiment servant de magasin, a été aménagé un petit laboratoire pourvu des instruments nécessaires aux analyses chimiques essentielles et à toutes les investigations hydrobiologiques.

Comme tous łes autres étangs de Zdencina, ceux affectés aux recherches sont installés sur un sol forestier défriché, constitué essentiellement par une argile brune aux teintes variées. La couche imperméable, sise à la profondeur, relativement grande, de 2 M. $58-2$ M. 70 , est formée d'une argile lourde, compacte, de couleur vert-noirâtre, ferrugineuse et riche en matières organiques. Est à noter l'absence complète de chaux dans tous les échantillons examinés.

Les travaux de la petite et modeste Station de Zdencina ont porté jusqu'ici sur les objets suivants :

$I^{\circ}$ Etat chimique (alcalinité, acidité ionique; teneur en oxygène et en acide carbonique, dureté) ;

$2^{\circ}$ Etat thermique (à la surface et en profondeur);

$3^{\circ}$ Etat biologique (plancton, benthos);

$4^{\circ}$ Travaux d'amélioration. (Amendements : - Superphosphate à $\mathrm{I} 6 \%$, kaïnite à $40 \%$, chlorure de potassium, vase de pêcherie, fumier d'étable. - - Faucardement et enlèvement de plantes nageanles, en particulier de la Châtaigne d'eau (Trapa natans) dont la végétation est, en Yougoslavie, luxuriante).

Des essais de nourrissage ont dû être abandonnés, car les agents de l'Institut de biologie appliquée ne peuvent venir à Zdencina que deux fois par mois, ces essais insuffisamment contrôlés n'ont donné aucun résultat notable.

Les principales constatations relatives à l'exploitation des étangs ont été, pour les cinq premières années d'existence de la Station, celles ci-après sommairement exposées.

Alcalinité. - Dans la règle, au début de la saison carpicole, donc en Avril-Mai, l'alcalinité est relativement forte, comme conséquence du chaulage pratiqué préalablement à l'empoissonnement, à la dose moyenne de goo kilogrammes par Hectare. Mais elle diminue sensiblement par la suite, surtout dans les étangs ayant reçu, soit du superphosphate, soit une fumure, où elle est toujours plus faible que dans ceux non amendés ; la réduction est, au surplus, proportionnelle aux quantités d'engrais apportées. Enfin, l'alcalinité recommence à croître, en Septembre, dans les étangs ayant reçu en Aout la quatrième et dernière distribution de superphosphate.

Les conditions d'alimentation en eau influent aussi sur l'alcalinité, qui varie en sens inverse du débit du canal d'amenée.

Concentration des ions-hydrogène. - L'action des amendements s'exerce sur l'acidité réelle, plus faible, en moyenne, là où il en a été pratiqué. 
On observe aussi que la valeur de $\mathrm{pH}$ dépend de la vitesse de renouvellement de l'eau.

Oxygénation. - L'influence est manifeste de la végétation nageante sur la teneur de l'eau en oxygène, spécialement celle de la Châtaigne d'eau, qui, en Croatie, couvre souvent, en un temps relativement court, la surface entière des étangs.

Au printemps, tant que la plante n'est pas encore apparue à la surface, la quantité de gaz respirable dissous est considérablement plus élevée qu'en été, où elle diminue au fur ot à mesure du développement de la Châtaigne pour croître ensuite, en Aoùt et Septembre, quand cette dernière commence à dépérir. La relation devient plus nette encore en cas de faucardement. La teneur en oxygène est notablement supérieure dans les étangs fauchés, surtout dans ceux complètement débarrassés des Plantes coupées.

L'effet des amendements est scnsible ; les étangs en ayant bénéficié accusent une teneur moindre que les autres, la réduction enregistrée étant proportionnelle à l'importance des apports.

Acide carbonique. - Il est habituel que la quantité d'acide carbonique libre soit plus faible dans les étangs amendés ; la plupart du temps ellẹ est en relation directe avec l'alcalinité et en sens inverse de l'oxygénation.

Il en vą de même de l'acide carbonique combiné qui diminue avec l'importance des quantités d'engrais. Sa teneur est, par ailleurs, à l'opposite de celle en acide libre.

Dureté. - La dureté totale est maxima au printemps, en raison du chaulage, et toujours plus élevée dans les étangs amendés.

Température. - L'état thermique, à l'instar de l'oxygénation, est étroitement dépendant du développement dẹ la flore nageante. En particulier, Jit différence entre la température superficielle et celle du fond peut atteindre $2^{\circ} \mathrm{C}$. et au delà quand le tapis flottant de Châtaignes d'eau est ininterrompu. Dans les étangs faucardés, l'écart est toujours plus faible, et s'atténue d'autant plus que les plantes ont été plus complètement éliminées.

Plancton. - Les recherches relatives au plancton ont montré que les viviers de recherches étaient de faible ou, au plus, de moyenne productivité. Ies quantités recueillies n'ont ordinairement pas atteint des chiffres élevés. Par contre, au point de vue qualitatif, le nombre des espèces dans les échantillons examinés a toujours été relativement grand.

L'effet des engrais s'avère notable, celui surtout du superphosphate qui, selon les conditions thermiques et métérologiques, provoque un accroissement de $\mathrm{I}_{7}$ à $30 \%$. Les autres amendements n'ont que peu ou pas d'effet.

Le comptage des espèces planctoniques conduit à des conclusions similaires.

Le faucardement, comme aussi l'enlèvement des plantes coupées, favorise le développement des microorganismes, la démonstration en est fournie par les examens quantitatifs ou qualitatifs du plancton : Qu'il s'agisse de la teneur globale ou de la variété des types, les étangs dont la végétation 
superficielle a été enlevée partiellement ou, mieux encore, totalement, l'emportent sur ceux où elle a été laissée après la coupe.

Benthos. - Les observations faites sur le plancton sont à étendre à la faune du fond des étangs. Même en $19^{27}$, où le développement de cette faune a été proportionnellement très fort, les constatations faites n'ont pas été à l'encontre, car cette abondance a été la conséquence d'une suite de circonstances météorologiques éminemment favorables, marquant plus spécialement lour empreinte sur la population benthique.

Le nombre des espèces composant cette dernière est toujours relativement faible.

Encore que l'influence des engrais ne soit pas aussi sensible que sur le développement du plancton, cependant clle se révèle par un accroissement d'environ $22-24 \%$. Ie superphosphate surtout provoque rette exhaustion. les autres engrais usucls n'ayant, ici encore, aucun effet appréciable. A noter, cependant, que le fumier d'étable, en $\mathrm{r}^{2} 7$, se montra plus actif que le superphosphate, car un étang en ayant reçu fut trouvé de $27 \%$ plus riche en organismes limicoles cu'un autre non fumé. Is nombre des espèces benthiques sélève aussi dans les étangs amendés.

Quand à l'effet du faucardement ct de l'enlèvement de la végétation aquatique superficiclle, jusqu'ici dans l'ensemble, il n'a pas été sensible. On remarque, toutefois, que la plupart du temps, la faune du fond est très pauvre dans les étangs non fauchés.

Production poissonnière. - Avant de résumer les constatations les plus importanies pour la pratique piscicole, à savoir celles concernant le rendement en Poissons, il doit être signalé que les étangs de recherches ont trujours été peuplés, moitié en Carpes à écailles, moitié en Carpes à miroirs, à raison, par Hectare, de 500 feuilles (I été) ou 200 nourrains (2 étés).

Au cours des cinq années rg?i-ig?g. l'accroissement, par Hectare, a varié, dans les étangs non amendés de ro $\bar{\jmath} \mathrm{K}$. ог̃o à $38_{2} \mathrm{~K}$. 820, dans ceux ayant reçu des engrais de 108 X. 2 Io à 570 K. 37o. Les meilleurs résultats ont été obtenus avec des doses de 3oo $\mathrm{K}$. de superphosphate par Hectare : celles de 200 ou r $50 \mathrm{~K}$. en donnant d'inférieurs. Avec les autres engrais, les rendements ont été encore moindres, le classement s'établissant ainsi : fumier d'étable ; - chlorure de potassium ; - vase de pêcherie ; - kaĩnite.

La production poissonnièrc d'étangs amendés est, en moyenne, supérieure de $40,37 \%$ à celle des étangs naturels. La majoration s'élève à $52,07 \%$ si on compare à ces derniers étangs ceux ayant reçu du superphosphate à $\mathbf{r} \%$, en quatre distributions égales, espacées de Mai à Août.

$\Pi$ est à remarquer que le rendement le plus 'élevé a toujours été obtenu э vec $300 \mathrm{~K}$. de superphosphate à l'Hectare, - soit avec un apport de $16 \mathrm{~K}$. d'acide phosphorique pur, - ce movennant quoi on a atteint un excédent moyen de $7 \mathbf{1}, 75 \%$ qui, pour $200 \mathrm{~K}$., flérhit à $15,5 \mathrm{I} \%$ et, pour $150 \mathrm{~K}$., à $\mathrm{I}, 35 \%$. 
En donnant $300 \mathrm{~K}$. à l'Hectare, répartis en deux apports seulement. au lieu daugmenter, le rendement reste inférieur à celui de l'étang non amendé.

Les mêmes résultats ont été obtenus avec rīo $\mathrm{K}$. de superphosphate et r $50 \mathrm{~K}$., soit de kaïnite à $40 \%$, soit de chlorure de potassium. Le superphosphate était distribué à quatre reprises, le complément à deux reprises seulement.

Un épandage de r.600 K. de vase de pècherie, effectué en quatre fois, provoqua un accroissement de $29,48 \%$, mais attribuable sans doute, au moins pour partie, à une action à retardement du superphosphate de l'année précédente (3(n) K.). Iutrement, on s'expliquerait mal que, pour l'ensemble des cinq années, l'apport de vase ait entraìné un rendement inféricur ì celui des élaugrs non amendés.

Un apport de sio K. de fumier d'étable, à la fin de Mai n'a donné qu'uI accroissement de $3.30 \%$.

L'influence du faucardement sur le rendement est évidente, car, dans les étangrs amendés et faucardés, l'augmentation, pour reux qui sont faurhés, s'élève à $152,55 \%$, chiffre remarquablement rilevé. Et. pour les étangs non amendés faucardés, rette augmentation est encore toujours supérieure à $9,20 \%$, alors que ceux non amendés, ni faucardés ont une production moyenne inférieure de $28,57 \%$ à celle des autres. Ft si on compare les étangs amendés non faucardés à ces derniers. on enregistre un accroissement d'environ $39,25 \%$.

Ces résultats mottent en relief le profit considérable qu'on retire, d'abord de l'engraissement des étangs, surtout en utilisant Ie superphosphate, puis de leur bon entretien par enlèvement de la végétation superficielle. Sans doute est-jl établi que l'amendement aver nourrissago concomitant élève considérabiement le rendement. mais les résultats obtenus à 7 dencina avec les seuls amendements fournissent une nowvelle prewve que le recours aux fertilisants, notamment au superphosphate, est beaucoup moins coûteux et, par suite, plus avantageux que le nourrissage. Fn outre, les Carpes encraissées, surfout an Maïs, sont trop grasses et de chair molle, moindre en sapidité.

Mentionnons encore, en terminant, que le rendement poissonnier a toujours été plus grand dans les étangs à riche faune limicole, co qui ne siobserve pas toujours à l'égard du plancton : il s'on déduit que les Carpes, dès le début du deuxième été, vivent surtout au dépens des organismes henthiques. En ouire, pour le type à écailles, le déchet a toujours été plus faible que pour la variété à miroirs : par contre, les sujets de rette dernière ont, d'ordinaire, un accroissement plus rapide.

Ce bref exposé des observations les plus saillantes faites à la Station yollgoslave de recherches carpicoles montre que les travaux qui y ont été poursuivis, confirment, sur la plupart des points, les résultats déjà acquis en matière d'exploitation des étangs. 